\title{
Increasing the Accuracy and the Repeatability of Position Control for Micromanipulations Using Heteroscedastic Gaussian Processes
}

\author{
Yanyu $\mathrm{Su}^{1}$, Wei Dong ${ }^{1}$, Yan $\mathrm{Wu}^{2}$, Zhijiang Du*1, Yiannis Demiris ${ }^{3}$
}

\begin{abstract}
Many recent studies describe micromanipulation systems by using complex Analytic Forward Models (AFM), but such models are difficult to build and incapable of describing unmodelable factors, such as manufacturing defects. In this work, we propose the Enhanced Analytic Forward Model (EAFM), an integrated model of the AFM and the Heteroscedastic Gaussian Processes (HGP). The EAFM can compensate the shortfalls of the AFM by training the HGP on the residual of the AFM. This also allows the HGP to learn the repeatability of the micromanipulation system. Based on the EAFM, we further contribute an optimal position controller for improving the accuracy and the repeatability. This optimal EAFM controller is implemented and tested on a three degreeof-freedom micromanipulator based micromanipulation system. Two sets of real-world experiments are carried out to verify our method. The results demonstrate that the controller using EAFM can statistically achieve higher accuracy and repeatability than solely using the AFM.
\end{abstract}

\section{INTRODUCTION}

Micromanipulation systems (shown in Fig.1a), which usually consist of a tool and a micromanipnulator, have been studied in various contexts. So far, increasing the accuracy and the repeatability of micromanipulation position controllers still remains as a challenge. Three main kinds of controllers are reported on this topic: 1) The feedback controllers [1], which use feedback sensors, are model-free but have the drawback of phase lag to apply in applications requiring high-frequency response; 2) The feed-forward controllers [2], which generally construct a feed-forward compensation scheme after building a model of the system, do no have phase lag but need a very accurate model; 3) The feed-forward approach has also been used with feedback approaches to harness the advantages of both [3].

Building forward/inverse models of micromanipulation systems is the first step towards designing a controller for micromanipulation systems. Various analytic models along with parameter identification methods, such as [4], [5], [6], have been investigated on this topic. However, such methods are unable to describe the unmodelable factors, such as manufacturing defects of the flexure hinge as illustrated in Fig. 1b. A promising solution for this problem is to learn

\footnotetext{
${ }^{1}$ Yanyu Su, Wei Dong and Zhijiang Du are with the State Key Laboratory of Robotics and System, Harbin Institute of Technology, China. suyanyu, dongwei, duzj01 at hit.edu.cn

${ }^{2}$ Yan $\mathrm{Wu}$ is with the Institute for Infocomm Research, Agency for Science, Technology and Research, Singapore. wuy at i2r.a-star.edu.sg

${ }^{3}$ Yiannis Demiris is with the Department of Electrical \& Electronic Engineering, Imperial College London, United Kingdom. y.demiris at imperial.ac.uk
}

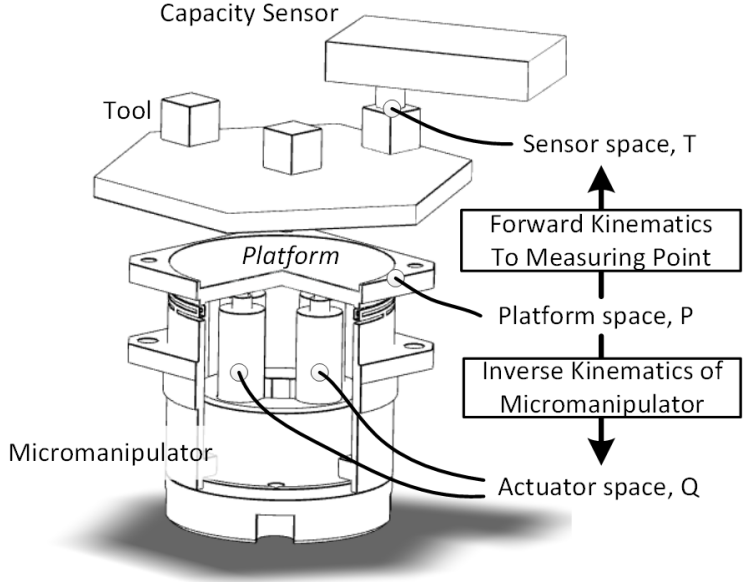

(a)

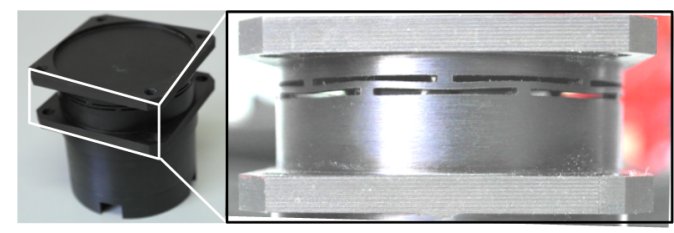

(b)

Fig. 1: The micromanipulation system of this work is shown in (a). The system consists of a micromanipulator, a calibration block and a capacitance gauge. The micromanipulator adjusts the mounted calibration block to control the distance between the measurement point and the capacitance gauge. (b) shows the inevitable manufacturing defects which introduce unmodelable uncertainty to the system resulting in the reduction of the accuracy and the repeatability of the micromanipulator.

the models of micromanipulation systems using machinelearning methods.

There are at least two directions of research to learn the models of the micromanipulation systems: 1) model-free methods which learn the systems without any prior knowledge [7]; 2) model-based methods which learn the residual of an analytic models [8]. Although the first approach produced some successful examples, its learning complexity scales drastically with the number of actuators involved. On the other hand, to our knowledge, no literature uses the latter approach considering the repeatability of micromanipulation systems.

In this paper, we present an Enhanced Analytic Forward Model (EAFM) to model a micromanipulation system using a combination of the Heteroscedastic Gaussian Processes (HGP) and the Analytic Forward Model (AFM). To build the EAFM, we use the HGP to model the residual between 
the prediction of the AFM and the feedback data. Unlike other machine learning methods either predicting points (e.g. Supported Vector Machine) or providing the predictive mean and distribution of a consistent variance (e.g. Gaussian Processes), the HGP predicts the mean and distribution of a possibly different variance at each point of problem space. Therefore the HGP is an apt tool for modelling the mean and the variance of the residual, which represent the accuracy of the AFM and the repeatability of the micromanipulation system respectively. In the EAFM, the output mean of the HGP is used to compensate the AFM on-the-fly while the variance is used to indicate the system repeatability.

We also address the optimal control problem of obtaining maximum repeatability using the EAFM. If the micromanipulation system is redundant, it is possible to reach the desired pose of the tool with different configurations. Given the initial state and the desired pose of the tool, we apply an optimal control approach to find the optimal control signal against the variance. Limited studies on micromanipulation consider this problem. An approach with similar goal solely uses analytic model to determine the best control policy to gain high repeatability for industrial manipulators [9]. However, this method relies heavily on the analytic model being accurate in order to identify the repeatability.

The rest of the paper is organised as follows: In Section II we will present the proposed EAFM and the optimal controller, followed by the application of this method on a micromanipulation system described in Section III. We will present the kinematic model of the micromanipulator and implement the method on a simple calibration system containing this micromanipulator. Two sets of experiments and their results are detailed and discussed in Section IV.

\section{Methodology}

In this work, we consider the problem of position control of the micromanipulation systems as shown in Fig.1a. We assume the controller of the micromanipulator takes the input of the pose (combination of position and orientation) of the platform. We also assume the state of the micromanipulation system is fully observable for training purpose.

\section{A. Definitions}

We model the micromanipulation system as a discrete-time system in the form

$$
\hat{s}_{k+1}=f\left(s_{k}, u_{k}\right)+\varepsilon\left(s_{k}, u_{k}\right),
$$

where $u_{k}$ denotes the current control signal defined by

$$
u_{k}=p_{k},
$$

with $p_{k} \in R^{n_{p}}$ denoting the current pose of the platform ( $n_{p} \in\left[1, n_{p}\right]$ denoting the degree of freedom (DoF) of the platform), $s_{k}$ denotes the state of the micromanipulation system at current time step

$$
s_{k}=\left[\begin{array}{ll}
t_{k}^{T} & q_{k}^{T}
\end{array}\right]^{T}
$$

which consists of the controlled pose of the tool $t_{k} \in R^{n_{t}}$ ( $n_{t} \in\left[1, n_{p}\right]$ denotes the DoF of the tool) and the displacements of the actuators $q_{k} \in R^{n_{q}}$ ( $n_{q}$ denotes the number of actuators), $\hat{s}_{k+1}$ denotes the prediction of successor state. The transition function $f\left(s_{k}, u_{k}\right)$ and the noise function $\varepsilon\left(s_{k}, u_{k}\right)$ respectively predict the successor state and noise from the current state-action pair $\left(s_{k}, u_{k}\right)$.

\section{B. Learning the Transition Function and the Noise Function}

In practice, the transition function is normally placed by an AFM of the micromanipulation system (i.e. $f\left(s_{k}, u_{k}\right)=$ $f_{a}\left(s_{k}, u_{k}\right)$ with $f_{a}\left(s_{k}, u_{k}\right)$ denoting the AFM) and, the noise function is normally assumed to follow a constant Gaussian distribution (i.e. $r\left(s_{k}, u_{k}\right) \sim \mathscr{N}(0, \Sigma)$ with $\Sigma$ denoting a constant diagonal matrix of the noise variance). However, there are two limitations to these settings: the predictions of the AFM usually have significant errors due to the unmodelable factors and, the noise usually does not follow the constant Gaussian distribution noise model.

To overcome these limitations, we propose the EAFM by respectively placing the transition function and the noise function with

$$
\begin{aligned}
& f\left(s_{k}, u_{k}\right)=f_{a}\left(s_{k}, u_{k}\right)+g_{e}\left(s_{k}, u_{k}\right) \\
& \varepsilon\left(s_{k}, u_{k}\right) \sim \mathscr{N}\left(0, r_{e}\left(s_{k}, u_{k}\right)\right)
\end{aligned}
$$

where the function $g_{e}\left(s_{k}, u_{k}\right)$ predicts the residual of the AFM, the function $r_{e}\left(s_{k}, u_{k}\right)$ describes the variance of the noise. The noise function of EAFM is assumed to follow a Gaussian distribution with zero mean and variable variance, since ideally the residual of the EAFM should be zero but the variance indicating the repeatability of the system could be different at different states-action pairs. Therefore, by regressing the functions $g_{e}\left(s_{k}, u_{k}\right)$ and $r_{e}\left(s_{k}, u_{k}\right)$, the system is precisely modelled in the form

$$
\begin{aligned}
& \hat{s}_{k+1}=f_{a}\left(s_{k}, u_{k}\right)+g_{e}\left(s_{k}, u_{k}\right)+\varepsilon_{e}\left(s_{k}, u_{k}\right) \\
& \varepsilon\left(s_{k}, u_{k}\right) \sim \mathscr{N}\left(0, r_{e}\left(s_{k}, u_{k}\right)\right)
\end{aligned}
$$

The underlying regression model should be capable of describing the value and the distribution. We propose to model the residual of the AFM with Heteroscedastic Gaussian Processes (HGP). Consider the regression task where the training dataset $D \equiv\left\{x \in R^{D}, y=y\left(x_{i}\right) \in R\right\}_{i=1}^{n}$ is modelled as the sum of a latent function $g(x)$ plus independent noise $\varepsilon_{i}$

$$
y\left(x_{i}\right)=g\left(x_{i}\right)+\varepsilon_{i} .
$$

A HGP is defined by placing a GP prior on the latent function $g(x)$ and Gaussian priors on the noise terms $\varepsilon_{i}$

$$
\begin{aligned}
& g(x) \sim G P\left(0, k_{f}\left(x, x^{T}\right)\right) \\
& \varepsilon_{i} \sim \mathscr{N}\left(0, r\left(x_{i}\right)\right)
\end{aligned}
$$

which is specified by the covariance function $k_{f}\left(x, x^{T}\right)$ and different possible variance $r\left(x_{i}\right)$ at each point $x_{i}$.

In contrast to other regression methods, HGP regression (HGPR) provides predictive distributions and is able to learn the output noise with possibly input-dependent inconstant variance from training data through likelihood maximisation. These features make HGPR attractive for learning the residual of the AFM, with the functions $g_{e}(s, u)$ and $r_{e}(s, u)$ in 


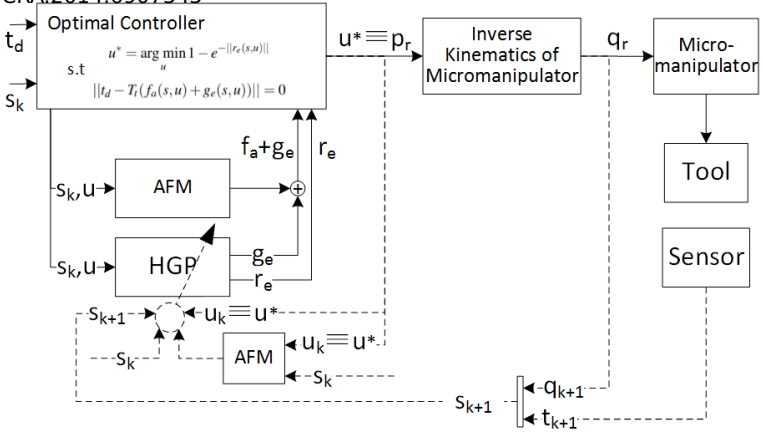

Fig. 2: The framework of the proposed controller for the micromanipulation. The dashed lines indicate the data flow of the learning process.

(1) corresponding to the function $g(x)$ and $r(x)$ in (2) and the training data defined by

$$
\begin{aligned}
& x=\left[\begin{array}{ll}
s^{T} & u^{T}
\end{array}\right]^{T} \\
& y=t^{\prime}-T_{t} f_{a}(s, u)
\end{aligned},
$$

where $x$ and $y$ are the input and output for training the HGP, $t^{\prime}$ is the measurements of the controlled pose of the tool after executing the control signal $u$, the matrix $T_{t}=\left[I_{n_{t} \times n_{t}} 0_{n_{t} \times n_{q}}\right] \in$ $R^{n_{t} \times\left(n_{t}+n_{q}\right)}$ transforms the state vector to the pose of the tool with $I_{n_{t} \times n_{t}}$ and $0_{n_{t} \times n_{q}}$ denoting the identity matrix and the zero matrix.

In the literature, alternative HGPR methods include the Markov chain Monte Carlo fully Bayesian method [10] which is very slow in large scale applications and the Maximum a Posteriori Approach [11] whose estimation does not integrate all latent variables and is prone to overfitting. In this work, we adopt the Variational Heteroscedastic Gaussian Process Regression (VHGPR) presented in [12], a variational inference method for HGPR based on variational Bayes and the Gaussian approximation, to learn and predict the residual of the AFM.

\section{Optimal Controller with EFAM}

Obtaining the maximum repeatability is a practically useful goal in position control of micromanipulator. With the EAFM, this goal can be considered as an optimal control problem to find the optimal control signal $u^{*}$ that minimizes the expected variance of the noise

$$
u^{*}=\underset{u}{\arg \min } 1-e^{-\left\|r_{e}(s, u)\right\|}
$$

subject to the constraint that the predicted error should be zero

$$
\left\|t_{d}-T_{t}\left(f_{a}(s, u)+g_{e}(s, u)\right)\right\|=0,
$$

where $t_{d}$ denotes the desired pose of the tool.

Therefore, we construct the control framework shown in Fig.2. Given the input of the desired pose of the tool $t_{d}$, the optimal controller generates the control signal $u^{*}$ (i.e. required pose of the platform $p_{r}$ ) by solving the optimal control problem defined in (4). Then the required movement of the actuators $q_{r}$ is accounted by solving the inverse kinematics of the micromanipulator subject to the required pose of the platform $p_{r}$. There are many optimization solvers to solve the optimal problem. In this work, we adopted the interior point algorithm [13].

\section{CONTROL OF A Microanipulator}

In this section, we apply the method presented in Section II on a micromanipulation system which consists of a micromanipulator, a calibration block and a one channel capacitance gauge, as shown in Fig. 1, to control the distance between the calibration block and the capacitance gauge.

\section{A. Inverse Kinematics of the Micromanipulator}

The structural design of the micromanipulator, shown in Fig.3b, consists of three parts: the Base, the PZTs and the Cover. The Base contains four slots for mounting the PZTs. On the bottom of these slots, four set-screws are used for adjusting the height of the PZTs to preload the contact force between the cover and the steel-balls located at the top of the PZTs. The cover integrates the flexure hinge and the platform for mounting the tools (e.g. the calibration block). The flexure hinge will be detailed later.

With the structural described above, the PZTs drive the platform by pushing the platform with the steel-balls. This transmission mechanism follows the contact model. We therefore apply the contact analysis method described in [14] for the inverse kinematics of the micromanipulator under the following assumptions: 1) Every steel-ball contacts the platform at one and only one point, where there is a unique, well-defined tangential plane for defining the normal vector. 2) The motion of the platform is small so the deformation of the flexure hinge is elastic.

The coordinate systems used in this work are illustrated in Fig.3c: 1) The world frame $\{\mathbf{N}\}$ is an inertial frame whose origin is conveniently chosen at the geometric center of the flexure hinge. All quantities are expressed in the world frame unless otherwise specified. 2) The platform frame $\{\mathbf{P}\}$ is fixed on the platform with its origin located at the origin of the world frame. 3) Let the steel-ball of the $i$-th PZT contact the bottom plane of the platform at contact point $i$ with $i=1,2 \ldots 4$ denoting the index of the PZTs. Let the vector $c_{i} \in R^{3}$ represents the position of the contact point $i$, which can be approximately estimated using the Computer Aided Design (CAD) model of the micromanipulator. 4) The contact point $i$ is chosen as the origin of the contact frame $\{\mathbf{C}\}_{i}$ with the axes $\left\{n_{i}, o_{i}, a_{i}\right\}$, where the unit vector $n_{i}$ is defined normal to the contact tangential plane (the bottom plane of the platform) and direct towards the platform, while the selection of $o_{i}$ and $a_{i}$ is arbitrary.

The flexure hinge is manufactured by cutting two stagger rows of slits on the cover. This structure can be simplified as a continuous beam supported by laterally and rotationally fixed supports. Since bending is the main mode of deformation for a continuous beam, this enables the platform to translate along $z$ axis and rotate around $x$ and $y$ axis in $\{\mathbf{N}\}$. The flexure hinge can therefore be simplified as a three-DoF hinge, which sits at the geometric center of the flexure hinge 


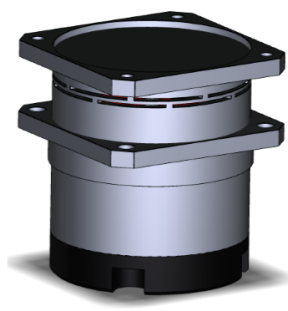

(a)

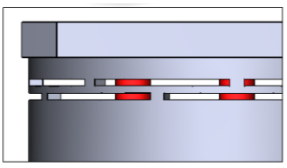

(b)

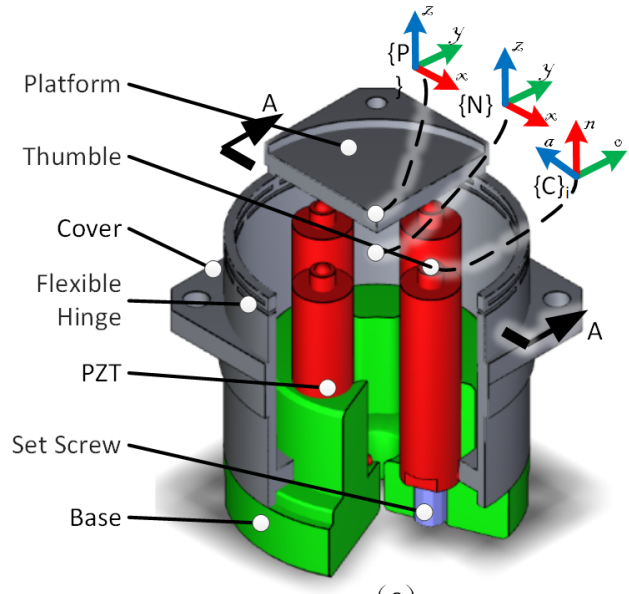

(c)

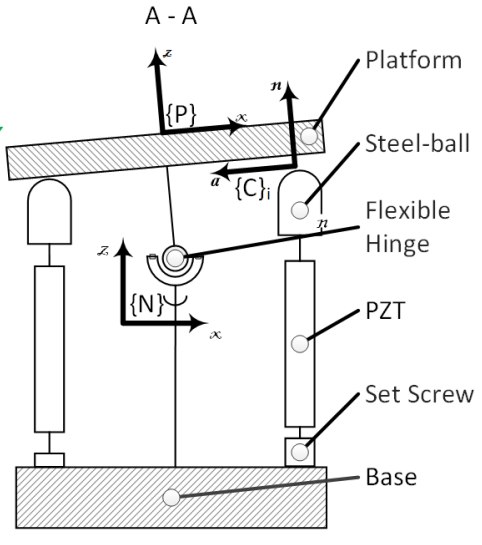

(d)

Fig. 3: Our micromanipulator is shown in (a) with its detailed structural design shown in (c). The micromanipulator consists of three parts: the Base (coloured in green), 4 PZTs (red) and the Cover (grey), where the novel flexure hinge shown in (b) located. The kinematic model of the manipulator is shown in (d). The flexure hinge is simplified as a three-DoF hinge and, the transmission mechanism between the steel-ball and the platform is described using the contact model.

with its motion described by $p=\left[z, r_{x}, r_{y}\right]^{T}$. More precise analysis on the flexure hinge can be made using elastic mechanics, however it is beyond this work.

With the equivalent hinge, we can build the kinematic model of the manipulator as shown in Fig.3d. Let $v=$ $\left[v^{T} \omega^{T}\right]^{T} \in R^{6}$ denote the twist of the platform, which is composed of the translational velocity $v \in R^{3}$ and the angular velocity $\omega \in R^{3}$. It is constrained by the flexure hinge,

$$
v=J_{h} \dot{p},
$$

where the vector $\dot{p}=\left[\begin{array}{l}\dot{z} \\ \dot{r_{x}} \\ \dot{r_{y}}\end{array}\right]^{T}$ is the velocity of the flexure hinge, the matrix $J_{h}=\left[\begin{array}{lll}0_{3 \times 2} & I_{3 \times 3} & 0_{3 \times 1}\end{array}\right]^{T} \in R^{6 \times 3}$ transforms $\dot{q}$ into the twist of the flexure hinge. Therefore, the platform has three actual DoF and, the motion of the flexure hinge can be adopted to describe the motion of the platform.

The twist of the platform $v$ is mapped from the world frame $\{\mathbf{N}\}$ to the $i$-th contact frame $\{\mathbf{C}\}_{i}$ by

$$
v_{i, p}^{C}=\bar{R}_{i}^{T} P_{i}^{T} v,
$$

where $v_{i, p}^{C} \in R^{6}$ denotes the twist of the platform expressed in the $i$-th contact frame $\{\mathbf{C}\}_{i}, P_{i} \in R^{6 \times 6}$ is the matrix

$$
P_{i}=\left(\begin{array}{cc}
I_{3 \times 3} & 0 \\
S\left(c_{i}\right) & I_{3 \times 3}
\end{array}\right),
$$

which relates the twist of platform $v$ and the twist of the $i$-th Contact Point on the Platform (CPP) with the cross-product function $S$ given a three-element vector $r=\left[\begin{array}{lll}r_{1} & r_{2} & r_{3}\end{array}\right]^{T}$,

$$
S(r)=\left(\begin{array}{ccc}
0 & -r_{3} & r_{2} \\
r_{3} & 0 & -r_{1} \\
-r_{2} & r_{1} & 0
\end{array}\right),
$$

the matrix $\bar{R}_{i} \in R^{6 \times 6}$ translates the twist of the CPP from the world frame $\{\mathbf{N}\}$ to the $i$ th contact frame $\{\mathbf{C}\}_{i}$,

$$
\bar{R}_{i}=\operatorname{Blockdiag}\left(R_{i}, R_{i}\right)=\left(\begin{array}{cc}
R_{i} & 0 \\
0 & R_{i}
\end{array}\right),
$$

where matrix $R_{i}=\left[n_{i} o_{i} a_{i}\right] \in R^{3 \times 3}$ is the orientation of the $i$ th contact frame $\{\mathbf{C}\}_{i}$ and, the function Blockdiag constructs block diagonal matrix from arguments of matrices.

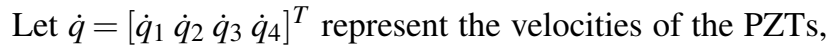
where $\dot{q}_{i} \in R$ denotes the velocity of the $i$-th PZT. It is related to the twist of the $i$-th Contact Point on the Steel-ball (CPS) with the equation

$$
v_{i, t}^{C}=\bar{R}_{i}^{T} J_{i} \dot{q},
$$

where $v_{i, t}^{C} \in R^{6}$ is the twist of the $i$-th CPS expressed in the $i$-th contact frame $\{\mathbf{C}\}_{i}, J_{i} \in R^{6 \times 4}$ is the Jacobian that maps the velocities of the PZTs to the twist of the $i$-th CPS, which is also translated from the world frame $\{\mathbf{N}\}$ to the respective contact frame $\{\mathbf{C}\}_{i}$ by left multiplying the matrix $\bar{R}_{i}{ }^{T}$.

Since the contact patch between the platform and each steel-ball is small and slippery, in this work, we adopt the frictionless point-contact model for the contact constraints between the platform and the steel-balls. With this model, only the normal component of the translational velocity of the contact point on the stee-lball is transmitted to the platform, that is,

$$
H_{i}\left(v_{i, t}^{C}-v_{i, p}^{C}\right)=0,
$$

where $H_{i}=\left[\begin{array}{llllll}1 & 0 & 0 & 0 & 0 & 0\end{array}\right]$.

It is practical to substitute (6), (7) into (8) and stack into a compact notation

$$
H\left(\bar{R}^{T} J \dot{q}-\bar{R}^{T} P^{T} v\right)=0,
$$

where

$$
\begin{aligned}
H & =\operatorname{Blockdiag}\left(H_{1}, H_{2}, H_{3}, H_{4}\right) \\
\bar{R}^{T} & =\operatorname{Blockdiag}\left(\bar{R}_{1}{ }^{T}, \bar{R}_{2}{ }^{T}, \bar{R}_{3}{ }^{T}, \bar{R}_{4}{ }^{T}\right) \\
J & =\left[\begin{array}{llll}
J_{1}^{T} & J_{2}^{T} & J_{3}^{T} & J_{4}^{T}
\end{array}\right]^{T} \\
P^{T} & =\left[\begin{array}{llll}
P_{1} & P_{2} & P_{3} & P_{4}
\end{array}\right]^{T}
\end{aligned}
$$


When $H R^{T} J \in R^{4 \times 4}$ is invertible, the inverse kinematics is obtained from (9) in the form of

$$
\dot{q}=\left(\left(H \bar{R}^{T} J\right)^{-1} H \bar{R}^{T} p^{T}\right) v,
$$

where the twist of the platform $v$ has to fulfil the constraint defined in (5) or, equivalently we can substitute (5) into (10),

$$
\dot{q}=\left(\left(H R^{T} J\right)^{-1} H R^{T} p^{T}\right) P_{j}^{T} J_{h} \dot{p},
$$

which maps the twist of the flexure hinge to the velocities of the PZTs.

Since (10) is obtained using the screw theory, its results are also applicable for the infinitessimal motions,

$$
\delta q=\left(\left(H R^{T} J\right)^{-1} H R^{T} p^{T}\right) P_{j}^{T} J_{h} \delta p,
$$

where $\delta q=\left[\begin{array}{llll}\delta q_{1} & \delta q_{2} & \delta q_{3} & \delta q_{4}\end{array}\right]^{T} \in R^{4}$ and $\delta q=$

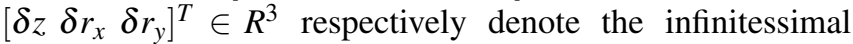
motions of the PZTs and the flexure hinge.

The displacement of the flexure hinge in a micromanipulation is small enough to be considered as an infinitessimal motion. So given the initial value $p_{0}$ and $q_{0}$, the required movement of PZTs for moving the flexure hinge to a desired pose is accounted for by solving (11) subject to $\delta q=q-q_{0}$ and $\delta p=p-p_{0}$. The initial state is normally set to zero, that is, $p_{0}=0$ and $q_{0}=0$, therefore,

$$
q=\left(\left(H R^{T} J\right)^{-1} H R^{T} p^{T}\right) P_{j}^{T} J_{h} p .
$$

\section{B. Implementation of the Controller}

The method presented in Section II is implemented and tested on our micromanipulator. The first prototype of our micromanipulator has some manufacturing defects, which makes it a very suitable application of the proposed method.

1) Observation of the pose of the Tool: We used a one channel capacitance gauge (i.e. Micro-Epsilon RS6500) to measure the movement of the tool (calibration block). The measurement range and the sensitivity of capacitance gauge is respectively $200 \mathrm{um}$ and and $0.0006 \mathrm{um}$. To maximally use the device to measure the movement of the calibration block, we choose a measurement point $50 \mathrm{~mm}$ away from the center of the platform.

2) AFM: Since the calibration block is mounted on the platform, the distance between the calibration block to the capacitance gauge is related to the pose of the platform by

$$
t=T_{s}\left(P_{t}^{T} J_{h} p+t_{0}\right),
$$

where $t \in R$ is the pose of the calibration block, the matrix $P_{t}$ maps the infinitessimal motion of the platform to the calibration block

$$
P_{t}=\left(\begin{array}{cc}
I_{3 \times 3} & 0 \\
S\left(t_{0}\right) & I_{3 \times 3}
\end{array}\right),
$$

and the matrix $T_{s}=\left[\begin{array}{lllll}0 & 0 & 1 & 0 & 0\end{array}\right]^{T}$ maps the pose of the calibration block to its distance to the capacitance gauge, $t_{0}$ is the initial pose of the calibration block. Therefore, by combining (12) and (13) the AFM is gained in the form

$$
s=\left[\begin{array}{c}
t \\
q
\end{array}\right]=\left[\begin{array}{c}
T_{s}\left(P_{t}^{T} J_{h} p+t_{0}\right) \\
\left(\left(H R^{T} J\right)^{-1} H R^{T} p^{T}\right) P_{j}^{T} J_{h} p
\end{array}\right] \in R^{5} .
$$

with parameters listed in Tab.I.
TABLE I: Parameters of the Implementation

\begin{tabular}{c|c|c|c}
\hline Symbol & Equation & Value & Unit \\
\hline$c_{1}$ & $(6)$ & {$[15,0,5]^{T}$} & $\mathrm{~mm}$ \\
\hline$c_{2}$ & $(6)$ & {$[0,-15,5]^{T}$} & $\mathrm{~mm}$ \\
\hline$c_{3}$ & $(6)$ & {$[0,15,0,5]^{T}$} & $\mathrm{~mm}$ \\
\hline$c_{4}$ & $(6)$ & {$\left[50 \cos \left(145^{\circ}\right), 50 \sin \left(145^{\circ}\right), 10\right]^{T}$} & $\mathrm{~mm}$ \\
\hline$t_{0}$ & $(14)$ & & $\mathrm{mm}$ \\
\hline
\end{tabular}

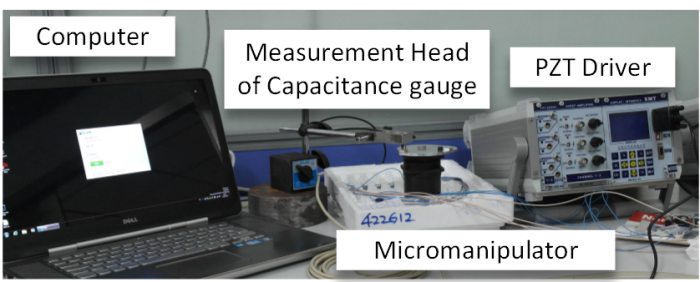

(a)

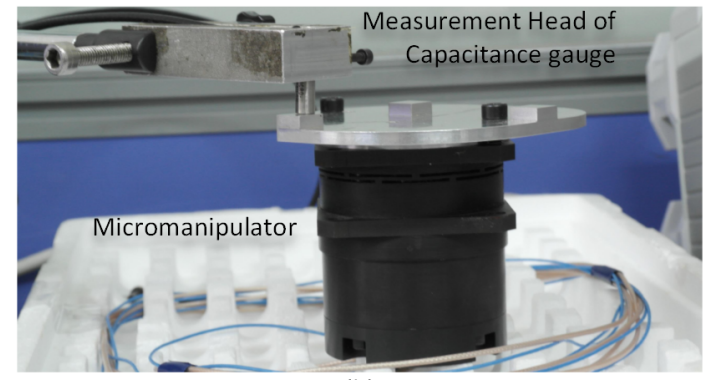

(b)

Fig. 4: The experiment environment is shown in (a). The host of the capacitance gauge is not put on the operation table therefore not shown in the figure. (b) details the probe of the capacitance gauge and the calibration block.

3) Position Control of PZTs: We used two PZT servo drivers to control the PZTs. Each driver has three channels (one channel for each PZT). The driver takes the input of the desired displacement and uses a PID controller to follow the input. The parameters of the drivers are tuned to the PZTs before the experiments. The accuracy of the driver is $0.01 \mathrm{um}$. The motion range of the PZTs is $0 \sim 38 \mathrm{um}$, but we took the range of $0 \sim 32$ um for safety reason.

\section{EXPERIMENTS AND RESULTS}

Referring to the general methods for testing the accuracy and the repeatability of a robot, we conducted two experiments to investigate: how the EAFM improves the accuracy over the AFM (the Absolute Movement Experiment) and how the proposed method improves the accuracy and the repeatability of the system (the Incremental Movement Experiment).

\section{A. Experiment Setup}

The experiment environment is shown in Fig. 4. Before training and the experiments, we measured the repeatability of the capacitance gauge, which is $\pm 1 \mathrm{um}$ in the experiment environment. We trained the HGP with 125 randomly generated control signals evenly distributed in the workspace before the experiments. 


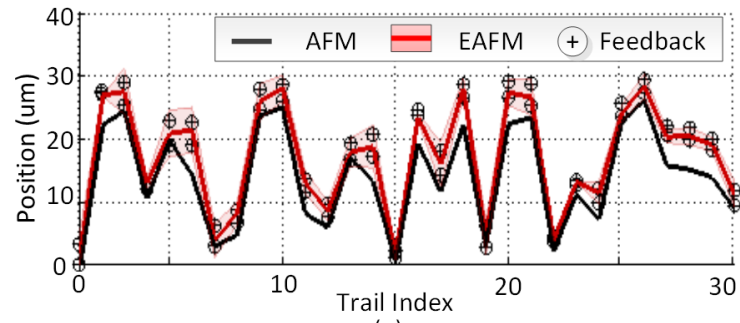

(a)

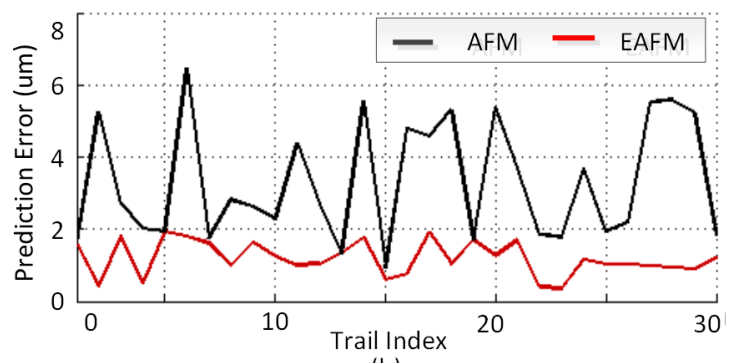

(b)

Fig. 5: The feedback and the predictions of the AFM and EAFM in the Absolute Movement Experiment are shown in (a). The solid red line is the predictive mean of EAFM (i.e. $g_{e}(s, u)$ ) and the light red area represents the predictive variance of EAFM (i.e. $g_{e}(s, u) \pm$ $\left.r_{e}(s, u)\right)$. The prediction errors of AFM and EAFM is shown in (b).

\section{B. Performance Evaluation Metrics}

We introduced four performance metrics to evaluate the performance of the controller quantitatively.

1) Prediction Error of Forward Model (PE): The prediction error of the EAFM $e_{m, e}$ and the AFM $e_{m, a}$ can be measured using the difference between their predictions and the feedback controlled pose of the tool

$$
\begin{aligned}
& e_{m, a}=\left|t^{\prime}-f_{a}(s, u)\right| \\
& e_{m, e}=\left|t^{\prime}-f_{a}(s, u)-g_{e}(s, u)\right|
\end{aligned}
$$

2) Mean of Steady-State Error (MSSE): The Steady-State Error (SSE) of the controller is a commonly used metric for the accuracy of controllers. In this paper, we calculate the SSE $e_{s}\left(t_{d}\right)$ at a desired controlled pose of the tool $t_{d}$ by

$$
e_{s}\left(t_{d}\right)=t_{d}-t_{f}
$$

where $t_{f}$ is the feedback of the controlled pose of the tool. To statically describe the result of the trials, we use the average of the SSE values at the same desired pose $e_{S}\left(t_{d}\right)$ to measure the accuracy of the controller.

3) Variance of Steady-State Error (VSSE): We use the variance of the SSE values at the same desired pose $e_{s}\left(t_{d}\right)$ to measure the repeatability of the controller.

4) Hypothesis Testing: To statistically evaluate the hypothesis that EAFM improves the performance of micromanipulation over AFM in terms of the accuracy and the repeatability, we applied the standard two-sample t-test on the MSSE and the VSSE of the EAKM controller against that of the AFM controller.
TABLE II: Statistical Results of Incremental Movement Experiment

\begin{tabular}{c|c|c|c|c|c|c}
\hline & Method & 7 um & 14 um & 21 um & 28 um & Unit \\
\hline MSSE & AFM & 1.2 & 3.1 & 3.0 & 5.2 & $\mu m$ \\
\hline MSSE & EAFM & 0.3 & 0.7 & 0.6 & 0.9 & $\mu m$ \\
\hline VSSE & AFM & 0.3 & 3.1 & 4.5 & 8.0 & $\mu m^{2}$ \\
\hline VSSE & EAFM & 0.14 & 0.15 & 0.5 & 3.5 & $\mu m^{2}$ \\
\hline
\end{tabular}

\section{Absolute Movement Experiment}

In this experiment, we check how the EAFM improves the prediction in the terms of accuracy. 30 control signals evenly covering the workspace, denoted by $u_{t}=\left\{u_{t, 1}, u_{t, 2} \ldots u_{t, 30}\right\}$, were randomly generated for conducting absolute movements from the initial position (i.e. $u_{t, 0}=[0,0,0]^{T}$ ), i.e. the platform was sequentially assigned to move the desired control signals of $u_{d}=\left\{u_{t, 0}, u_{t, 1}, u_{t, 0}, u_{t, 2}, \ldots u_{t, 0}, u_{t, 30}\right\}$ in each trial. These desired control signals are used to account for the commands sending to the PZTs drivers. 2 trials were conducted for each point.

The feedback value and the predictions of the AFM and EAFM are shown in Fig.5a. We can see that the feedbacks are all in the area specified by the predictive mean and variance of EAFM (i.e. $g_{e}(s, u) \pm r_{e}(s, u)$ ), which indicates that the EAFM can correctly describe the inaccuracy and the inconsistency of the system.

The PEs of both AFM and EAFM are shown in Fig.5b. We can see that the PEs of the EAFM are smaller than those of the AFM for all points. We further applied t-test respectively on the PEs of both models and, the result indicates that the PE of the EAFM is statistically smaller than that of the EAFM controller with significance level of $\alpha=0.05$ (P-value $\left.p=1 \times 10^{-6}\right)$.

\section{Incremental Movement Experiment}

In each trial of this experiment, 4 points $(7,14,21,28 \mathrm{um})$ at equal distance covering the workspace were selected for conducting continuous movements beginning from the initial position, that is, the controller was sequentially assigned to reach the desired positions of $t_{d}=\{0,7, \ldots 28\}$ in each trail. 10 trials were conducted in the experiment.

The SSE of both AFM and EAFM controllers are shown in Fig.6. We can see that the EAFM controller outperformed the AFM in nearly all trials. The only exception is the "28 um" in Trial 4.

The respective statistical results are shown in Tab. II. Both the MSSEs and the VSSEs of the EAFM controller are smaller than those of the AFM controller. We further applied t-test respectively on the ESSEs and VSSEs of both controllers, the result indicates that the ESSEs of the EAFM controller is statistically smaller than that of the AFM controller with significance level of $\alpha=0.05$ (Pvalue $p=0.01$ ) and the VSSEs of the EAFM controller is statistically smaller with significance level of $\alpha=0.05$ (Pvalue $p=0.07$ ). 


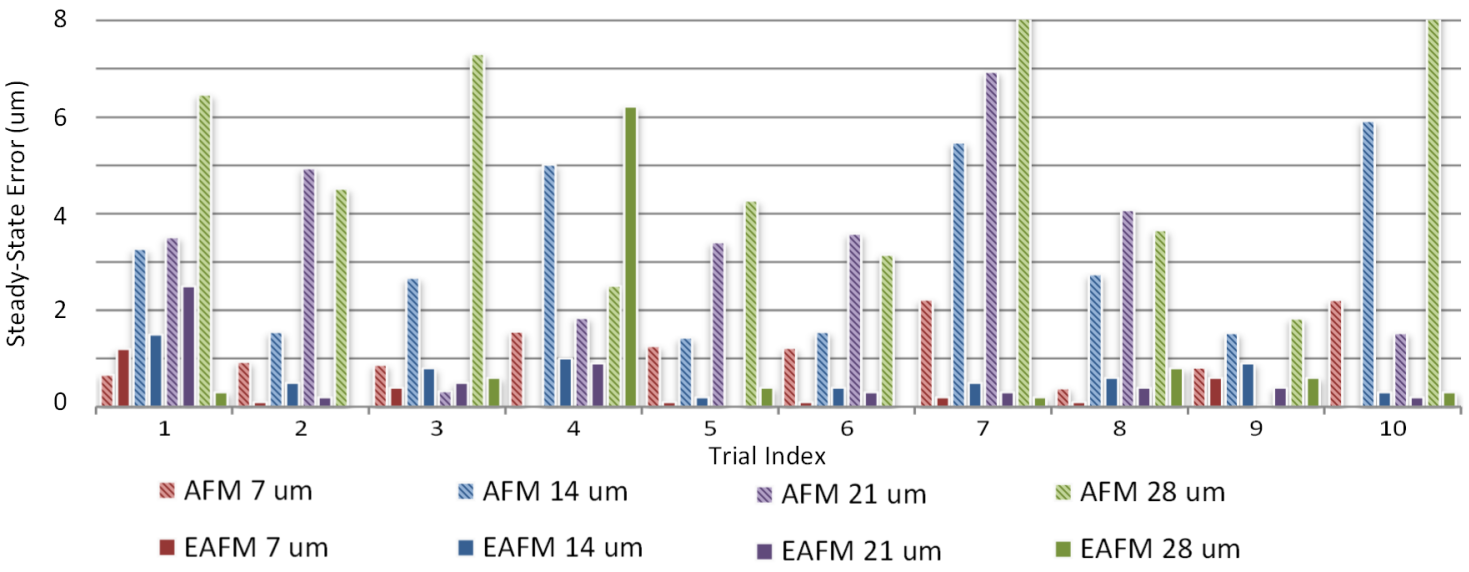

Fig. 6: The Steady-State Error of the controller using AFM and EAFM in the incremental movement experiment.

\section{CONCLUSION}

In this paper, we contributed an Enhanced Analytic Froward Model (EAFM) for a micromanipulatioin system, an integrated model of the Analytic forward Model (AFM) and the Heteroscedastic Gaussian Processes (HGP). The EAFM can compensate the error of the AFM by training the HGP on the residual of the AFM. Our experimental results showed that the EAFM produces more accurate predictions over the AFM. The EAFM is also be capable to model the variance of the feedback measurements, which indicates the repeatability of the system. The EAFM is applied to an optimal controller to increase the accuracy and the repeatability of the micromanipulatioin system. Our experimental results show the optimal controller with EAFM is capable to increase the accuracy and the repeatability over the controller with AFM.

Looking forward, we envision several improvements that would increase the utility of the method. The method is currently applied on a position control which only needs one control step. We will investigate an optimal controller for trajectory tracking that needs multiple control steps and, the aim of the controller would be to find an optimal control policy minimizing the expected cumulative variance.

\section{ACKNOWLEDGMENT}

The authors acknowledge the comments and support from Dr. Harold Soh. This research is funded by the Chinese Advanced Armament Research Project of 12-th Five-year Plan (Grant No. 51320030201), the Fundamental Research Funds for Central Universities (HIT.NSRIF.2014061), the HIT Disruptive Innovation Program and the HIT Overseas Talents Introduction Program.

\section{REFERENCES}

[1] Y. Li and Q. Xu, "Estimation and PID Sliding Surface for Motion Tracking of a Piezo-Driven Micromanipulator," Control Systems Technology, IEEE Transactions on, vol. 18, no. 4, pp. 798-810, 2010.

[2] Y. L. Zhang, M. L. Han, M. Y. Yu, C. Y. Shee, and W. T. Ang, "Automatic hysteresis modeling of piezoelectric micromanipulator in vision-guided micromanipulation systems," Mechatronics, IEEE/ASME Transactions on, vol. 17, no. 3, pp. 547-553, 2012
[3] Q. Xu and Y. Li, "Dahl model-based hysteresis compensation and precise positioning control of an XY parallel micromanipulator with piezoelectric actuation," Journal of dynamic systems, measurement, and control, vol. 132, no. 4, p. 41011, 2010.

[4] W. Dong, L. N. Sun, and Z. J. Du, "Design of a precision compliant parallel positioner driven by dual piezoelectric actuators," Sensors and Actuators A: Physical, vol. 135, no. 1, pp. 250-256, 2007.

[5] R. C. Shi, W. Dong, and Z. J. Du, "Design methodology and performance analysis of application-oriented flexure hinges," Review of Scientific Instruments, vol. 84, no. 7, p. 75005, 2013.

[6] Z. Du, R. Shi, and W. Dong, "A Piezo-Actuated High-Precision Flexible Parallel Pointing Mechanism: Conceptual Design, Development, and Experiments," Robotics, IEEE Transactions on, vol. 30, no. 1, pp. $131-137,2014$.

[7] D. Caligiore, T. Ferrauto, D. Parisi, N. Accornero, M. Capozza, and G. Baldassarre, "Using motor babbling and Hebb rules for modeling the development of reaching with obstacles and grasping," in Proceedings of International Conference on Cognitive Systems (CogSys 2008), Karlsruhe, Germany, pp. E1-8, 2008.

[8] P. Hemakumara and S. Sukkarieh, "Non-parametric UAV system identification with dependent Gaussian processes," in Proceedings of International Conference on Robotics and Automation (ICRA 2011), Shanghai, China, pp. 4435-4441, IEEE, 2011.

[9] T. Shamir and Y. Yomdin, "Repeatability of redundant manipulators: Mathematical solution of the problem," Automatic Control, IEEE Transactions on, vol. 33, no. 11, pp. 1004-1009, 1988.

[10] M. Goldfarb and N. Celanovic, "Modeling Piezoelectric Stack ALctuator for Control of Micromanipulation," Control Systems, IEEE, vol. 17, no. June, pp. 69-79, 1997.

[11] K. Kersting, C. Plagemann, P. Pfaff, and W. Burgard, "Most Likely Heteroscedastic Gaussian Process Regression," in Proceedings of International Conference on Machine Learning (ICML 2007), Corvallis, OR, USA, pp. 393-400, 2007.

[12] M. Lázaro-Gredilla, M. Titsias, and L. Miguel, "Variational heteroscedastic Gaussian process regression," in Proceedings of International Conference on Machine Learning (ICML 2011), Bellevue, Washington, USA, pp. 393-400, 2011.

[13] R. H. Byrd, M. E. Hribar, and J. Nocedal, "An interior point algorithm for large-scale nonlinear programming," SIAM Journal on Optimization, vol. 9, no. 4, pp. 877-900, 1999.

[14] D. Prattichizzo and J. C. Trinkle, "Grasping" in Springer Handbook of Robotics, pp. 671-700, Springer, 2008. 